\title{
Home range of the mouse Phyllotis osilae in forest fragments of Polylepis subtusalbida with different habitat matrix
}

\author{
Romeo Rojas-Estrada ${ }^{1 *}$, Luis F. Aguirre', and Freddy Navarro Antezana' \\ ${ }^{1}$ Centro de Biodiversidad y Genética, Universidad Mayor de San Simón. Casilla 538, Cochabamba, Bolivia. Email: \\ romeorojasestrada@gmail.com (RR-E), laguirre@fcyt.umss.edu.bo (LFA), frenan@fcyt.umss.edu.bo (FNA). \\ *Corresponding author
}

\begin{abstract}
Home range is an aspect of ecology that allows us to understand the mechanisms and spatial relationships that underlie habitat choice and animal responses to environmental change. However, studies on this subject have been focused mainly on medium and large mammals, while those on small mammals are scarce. Small mammals play important roles in maintaining ecological processes, such as the survival of several plant species. Phyllotis osilae is a phyllotine rodent, which functions as a potential seed disperser for Polylepis subtusalbida, a tree that is endemic and categorized as Vulnerable (VU) for Bolivia. The forests of $P$. subtusalbida are highly fragmented, with reforestation with introduced species such as Pinus radiata and Eucalyptus globulus. The question we pose for this study is: how does the home range of $P$. osilae vary between fragments of $P$. subtusalbida with different types of matrix (natural grassland and exotic tree plantations)? The study was carried out on four fragments of $P$. subtusalbida in Tunari National Park in the city of Cochabamba, Bolivia: two fragments in the exotic tree plantation matrix (Pajcha locality) and two in the natural grassland matrix (San Miguel locality). For telemetry purposes, radio collars were installed on 16 individuals. The home ranges were calculated using the Minimun Convex Polygon (MCP) at $95 \%$, in the same way the Kernel density (KDE) was estimated at $95 \%$. The percentage of presence of $P$. subtusalbida and matrix trees within each home range was determined, with an area interval between $50.5 \mathrm{~m}^{2}$ and 3,010 $\mathrm{m}^{2}$. No significant differences were found between both matrices regard to the area of activity; however, in the comparison of the percentages of presence of $P$. subtusalbida trees and matrix, significant differences were found. Our results for home ranges show that the matrix would not have an effect on the area, but on its mobility; this is evidenced by the absence of movements of $P$. osilae from the fragments to the exotic matrix. In this case, the exotic tree matrix is operating as a barrier and is reflected in the almost total absence of this vegetation matrix in the home range. P. osilae may be playing an important role in the recovery and conservation of these ecosystems, so knowing aspects of their natural history as well as their responses to fragmentation is of great importance in understanding the fate of the Polylepis forests.
\end{abstract}

El ámbito hogareño es un aspecto de la ecología que permite comprender los mecanismos y las relaciones espaciales que fundamentan la elección del hábitat y las respuestas de los animales a los cambios ambientales. Sin embargo, los estudios de esta temática han sido enfocados principalmente a mamíferos medianos y grandes, en contraste los de pequeños mamíferos son escasos. Los pequeños mamíferos cumplen roles importantes para el mantenimiento de procesos ecológicos, como la sobrevivencia de varias especies de plantas. Phyllotis osilae es un roedor filotino, que funciona como potencial dispersor de semillas de Polylepis subtusalbida, un árbol endémico y categorizado como Vulnerable (VU) para Bolivia. Los bosques de P. subtusalbida están altamente fragmentados, presentando entre sus principales amenazas la reforestación con especies introducidas como Pinus radiata y Eucalyptus globulus. La pregunta que planteamos para este estudio es ¿cómo varía el ámbito hogareño de $P$. osilae entre fragmentos de $P$. subtusalbida con diferente tipo de matriz (pastizal natural y plantaciones de árboles exóticos)? El estudio se realizó en cuatro fragmentos de P. subtusalbida en el Parque Nacional Tunari de la ciudad de Cochabamba, Bolivia: dos fragmentos en la matriz de plantaciones de árboles exóticos y dos en la matriz natural de pastizal. Para la telemetría se instalaron radio collares a 16 roedores. Los ámbitos hogareños se calcularon mediante el Minimun Convex Polygon (MCP) al 95 \%, del mismo modo se estimó la densidad de Kernel (KDE) al $95 \%$. Se determinó el porcentaje de presencia de árboles de P. subtusalbida y matriz dentro de cada ámbito hogareño, con un intervalo de área entre $50.5 \mathrm{~m}^{2}$ a 3,010 $\mathrm{m}^{2}$. No se encontraron diferencias significativas entre ambas matrices respecto a el área de actividad; sin embargo, en la comparación de los porcentajes de presencia de árboles de $P$. subtusalbida y matriz, si se encontraron diferencias significativas. Nuestros resultados muestran que la matriz no tendría un efecto sobre el área, pero si sobre su movilidad. Esto se evidencia en la ausencia de movimientos de $P$. osilae de los fragmentos a la matriz exótica. En este caso, la matriz de árboles exóticos está operando como una barrera y se ve reflejado en la ausencia casi total de matriz de esta vegetación en el ámbito hogareño. P. osilae puede estar cumpliendo un rol importante en la recuperación y conservación de estos ecosistemas, por lo que conocer aspectos de su historia natural al igual que conocer sus respuestas a la fragmentación es de mucha importancia para comprender el destino de los bosques de Polylepis.

Keywords: endemic; fragmentation; Polylepis; telemetry.

(C) 2020 Asociación Mexicana de Mastozoología, www.mastozoologiamexicana.org

\section{Introducción}

El ámbito hogareño es el área utilizada por un individuo para buscar alimento, reproducirse, descansar y realizar todas sus actividades diarias (Burt 1943). Además, es un aspecto de la ecología que proporciona respuestas a muchas preguntas biológicas relacionadas con la dinámica de la población, las interacciones sociales, patrones de espaciamiento (Eiris y Barreto 2009) y distancia de dispersión (Santini et al. 2013). El análisis del ámbito hogareño es un primer paso importante para comprender los mecanismos y las relaciones espaciales que fundamentan la elección del hábitat y las respuestas de los animales a los cambios ambientales (Cumming y Cornélis 2012). Asimismo, el ámbito hogareño de hembras y machos pueden explicar 
distintos efectos del medio ambiente, debido a que en las hembras están relacionado con la distribución espacial de recursos y cobertura (Bonatto et al. 2012), mientras que para los machos dependen de la distribución espacial de las hembras (Ostfeld 1985). A la fecha, se carece de información sobre el ámbito hogareño para muchas especies o hábitats particulares (Wood et al. 2010). Tradicionalmente, los estudios de ámbito hogareño han sido enfocados principalmente a mamíferos medianos y grandes, y es muy difícil encontrarlos en pequeños mamíferos (Harris et al. 1990; Dressler et al. 2016).

Los pequeños mamíferos cumplen roles de importancia vital para el mantenimiento de procesos ecológicos, como la sobrevivencia de varias especies de plantas, la cual depende de la interacción de planta-animal que tiene efectos en la dispersión desde la planta madre, escarificación y enterramiento de las semillas (Tarifa y Aguirre 2009). Los roedores filotinos (Phyllotini Vorontsov, 1959) son pequeños mamíferos comunes en bosques de Polylepis (Yensen y Tarifa 2002). En este estudio, nos enfocamos en Phyllotis osilae, un roedor abundante en bosques Polylepis subtusalbida (Vargas et al. 2007; Tarifa y Yensen 2001) y a la vez un consumidor de semillas de esta especie (Balderrama 2013, com. pers.), por lo que su presencia y consumo de semillas de Polylepis lo hace un potencial dispersor.

Los bosques de Polylepis (Rosaceae, Sanguisorbeae) son bosques nativos altamente fragmentados a lo largo de su distribución; en Bolivia se ha estimado que ocupan el $10 \%$ de su distribución original (Fjeldså y Kessler 2004). Polylepis subtusalbida es una especie endémica para Bolivia y, según los criterios de la IUCN (Arrázola 2011) aplicados a la flora amenazada de Bolivia, está categorizado como Vulnerable (VU; Ministerio de Medio Ambiente, Agua y Cambio Climático 2011). Entre sus principales amenazas está la reforestación con especies introducidas como Pinus radiata y Eucalyptus globulus (Arrázola 2011). Estos árboles exóticos son parte ahora del estrato arbóreo donde están presentes los bosques de P. subtusalbida (Gareca et al. 2007), formando la matriz del paisaje de estos bosques fragmentados. En la ecología del paisaje, el modelo parche-corredor-matriz define a la matriz como el tipo de elemento dominante, más extensa y coherente del paisaje (Forman 1995). El tipo de matriz que rodea los hábitats fragmentados tienen efectos significativos sobre la biodiversidad en diferentes tipos de paisaje, escalas espaciales, enfoques ecológicos y grupos taxonómicos (Prevedello y Viera 2010). Los pequeños mamíferos que dependen del bosque, pero persisten en paisajes fragmentados, pueden ser utilizados como modelos para cuantificar los efectos de la pérdida y fragmentación del hábitat, y guiar las acciones de conservación para mejorar el bienestar de los animales en paisajes fragmentados (Delciellos et al. 2018).

Los mamíferos de los bosques de Polylepis han recibido poca atención científica (Yensen y Tarifa 2002) y la distribución de los animales asociados a estos bosques ha sido poco estudiada (Fjeldså y Kessler 2004). La falta de conocimiento sobre las especies de mamíferos en los bosques hace imposible comprender su historia natural, ecología y estado de conservación, siendo un hábitat altamente amenazado y fragmentado (Tarifa y Yensen 2001). Conocer las respuestas a la fragmentación de estos bosques nativos es esencial tanto para predecir su propio destino como para comprender el destino de los árboles que dependen de ellos (Jorge 2008). Por esta razón se evalúa la variación del ámbito hogareño de $P$. osilae entre fragmentos de $P$. subtusalbida con el pastizal natural y las plantaciones de árboles de Pinus radiata y Eucalyptus globulus.

\section{Material y métodos}

El estudio se realizó en cuatro fragmentos de P. subtusalbida en el Parque Nacional Tunari de la ciudad de Cochabamba, Bolivia (Figura 1): dos fragmentos se encuentran en la localidad de Pajcha con superficies de 10.7 ha y 3.6 ha

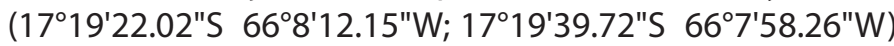
con una matriz de plantaciones de árboles exóticos compuesto por Pinus radiata y Eucalyptus globulus; dos fragmentos se encuentran en la localidad de San Miguel con superficies de 5.1 ha y 12.1 ha $\left(17^{\circ} 16^{\prime} 36.68^{\prime \prime S} .66^{\circ} 19^{\prime} 46.50^{\prime} \mathrm{W}\right.$; $17^{\circ} 16^{\prime} 27.30^{\prime \prime S}$. $\left.66^{\circ} 20^{\prime} 13.25^{\prime \prime W}\right)$ con una matriz natural de pastizales compuestos por Calamagrostis sp., Cheilanthes pruinata, Cyperus sp., Eryngium paniculatum, Festuca dolichophylla, Gamochaeta sp., Gnaphalium dombeyanum, Lupinus sp., Luzula sp., Puya tunariensis, Stevia samaipatensis y Stipa ichu. Ambas localidades se encuentran dentro el piso ecológico altimontano distribuidos en las laderas montañosas meridionales medias de la Cordillera del Tunari. La vegetación potencial natural del piso altimontano es dominado por $P$. subtusalbida, en los meses de mayo a agosto se presenta la época de sequía que coincide con el tiempo más frio $\left(-3\right.$ y $-6{ }^{\circ} \mathrm{C}$, respectivamente). De diciembre a febrero se presenta la época de lluvias que coincide con el tiempo cálido y se presentan precipitaciones anuales de 45 a $60 \%$ (Navarro et al. 2015).

El trabajo se realizó durante los meses de marzo a junio del 2013, terminando la época de lluvias y comenzando la época de sequia. Para la captura de $P$. osilae, se instalaron 160 trampas tipo Sherman para pequeños mamíferos en cada fragmento, siguiendo un protocolo de muestreo en micromamíferos altoandinos (Ruiz et al. 2004). Se realizaron líneas de trampeo, cada línea con 10 trampas y una separación de $10 \mathrm{~m}$. Se instalaron cinco líneas paralelas a los bordes, una al centro y dos a ambos lados del borde hasta una distancia de $20 \mathrm{~m}$ al interior del fragmento y la matriz. Las líneas de trampeo estuvieron activas de una a tres noches, sumando un esfuerzo de 480 trampas por fragmento. Como cebo se utilizó avena, esencia de vainilla, mantequilla de maní y uvas pasa. La revisión de las trampas y recebado se realizó cada 12 h. Una vez capturados los individuos necesarios para la realizar la telemetría se procedió a cerrar las líneas, para evitar recapturarlos. 


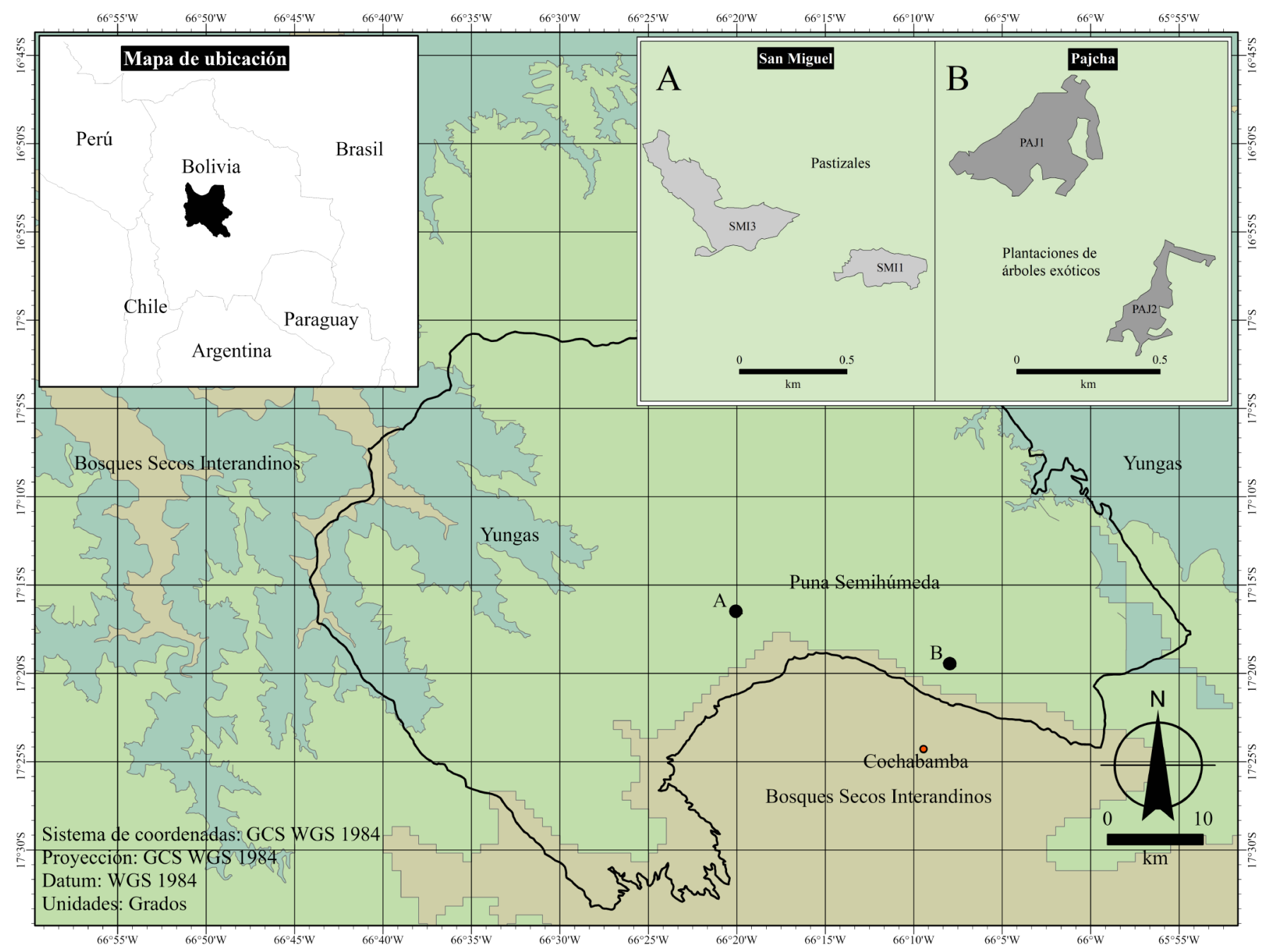

Figura 1. Mapa del área de estudio. Limites del Parque Nacional Tunari y subecoregiones presentes (Ibisch y Mérida 2003). Las ubicaciones de los fragmentos de Polylepis subtusalbida se indican con letras. A): Localidad de San Miguel con una matriz de pastizales, fragmentos SM13 y SM11. B): Localidad de Pajcha con una matriz de plantaciones de árboles exóticos compuesto por Pinus radiata y Eucalyptus globulus, fragmentos PAJ1 y PAJ2.

Para el manejo y manipulación de los individuos capturados se siguieron los lineamientos propuestos por Sikes et al. (2016), en concordancia con las leyes nacionales del Estado Plurinacional de Bolivia (Autorización MMAyAVMA-DGBAP $N^{\circ}$ 619/12). Los roedores se identificaron mediante claves (Hershkovitz 1962; Steppan 1995; Anderson 1997; Spotorno et al. 2004; Jayat y Pacheco 2006) y se registraron medidas morfométricas convencionales: longitud total, cola, pata posterior, oreja (Anderson 1997), también se registró el sexo, estado reproductivo, código numérico de los individuos, fragmentos de la matriz en donde se ubicaron sus ámbitos hogareños y el peso. El pesado de los individuos se obtuvo con una pesola de capacidad de $100 \mathrm{~g}$ y precisión de $\pm 0.3 \%$; la exactitud del peso de los individuos fue un factor determinante para la instalación de los transmisores.

Para la telemetría se instalaron radio collares VHF Telenax (TXB-004G) solamente a individuos en los que el peso del transmisor no superó en 5 a $10 \%$ el peso del animal (Sikes et al. 2011); para la instalación se siguieron las consideraciones y recomendaciones de White y Garrot (1990). El seguimiento de telemetría se realizó por nueve días en promedio, debido a la duración de la batería del transmisor, desde las 19:00 a 3:00 por los hábitos nocturnos que presenta P. osilae. El seguimiento no incluyó las primeras 24 horas tras liberar a los individuos, para permitir que se acostumbraran a los transmisores. Para el seguimiento se usó un receptor Telonics (TR-4K) y una antena Yagi de tres elementos, la posición se determinó mediante triangulación con un lapso de 60 minutos (en promedio) entre cada posición. Para cada posición se registraron sus coordenadas geográficas (punto fijo o fixed point). Se registraron datos adicionales de distancia y azimut entre cada punto fijo. Los ámbitos hogareños se calcularon en el programa BIOTAS Versión 2.0 Alpha (Ecological Software Solutions LLC) mediante el Polígono Mínimo Convexo (Minimun Convex Polygon, MCP) al $95 \%$ (Mohr 1947), también se estimó la densidad de Kernel (KDE; Worton 1989) al $95 \%$ para facilitar comparaciones.

Se determinó el porcentaje de presencia de árboles de P. subtusalbida y matriz dentro de cada ámbito hogareño de pastizal natural y plantaciones de $P$. radiata y E. globulus se sobrepuso una cuadricula sobre el área de actividad. Se utilizó una imagen satelital WorldView-2 (resolución espa- 
cial $0.5 \mathrm{~m}$ ) para la localidad de Pajcha y para la localidad de San Miguel una imagen satelital Maxar Technologies (resolución espacial $30 \mathrm{~m}$ ). En ambas imágenes satelitales fue posible identificar árboles de $P$. subtusalbida y la matriz. La fotointerpretación se realizó con los softwares de ArcGIS 10.2 (Esri, USA) y Google Earth Pro (Google LLC, USA). Se compararon las estimaciones de los ámbitos hogareños con el tamaño de muestra mediante una regresión lineal, con el software PAST Versión 4.03 (Hammer 2001), para determinar la independencia de las estimaciones. Se realizó un ANCOVA para comprobar las diferencias entre localidades para el área del ámbito hogareño y una prueba de $U$ de Mann-Withney para los porcentajes de presencia (árboles de Polylepis y matriz), ambos estadísticos fueron calculados mediante el software PAST Versión 4.03 (Hammer 2001).

\section{Resultados}

Capturamos tres especies de roedores para ambas localidades: Akodon boliviensis, Oxymycterus paramensis y Phyllotis osilae. Se monitoreó un total de 16 individuos de $P$. osilae, siete hembras y un macho en la localidad de Pajcha (matriz de árboles exóticos), y cinco hembras y tres machos en la localidad de San Miguel (matriz natural de pastizales). Las comparaciones entre las estimaciones de los ámbitos hogareños con el tamaño de muestra indica que no hay independencia $(r=0.775, t=4.5956, P=0.0004)$. Se estimó el área y perímetro de los ámbitos hogareños (Tabla 1; Figuras 2, 3), donde se obtuvo $3,010 \mathrm{~m}^{2}$ como el área máxima para la hembra $\mathrm{H} 2$ (Figura 3 ) y una mínima de $50.5 \mathrm{~m}^{2}$ para la hembra H5 (Figura 4). El área y perímetro núcleo (Kernel $95 \%$ ) no se obtuvo para todos los individuos debido al número limitado de puntos fijos. En promedio, se tuvieron nueve noches consecutivas de seguimiento, a excepción del macho M8, que después del tercer día se perdió la señal del transmisor (Tabla 1).

Dentro del área de los ámbitos hogareños de $P$. osilae se obtuvieron los porcentajes de presencia de árboles de P. subtusalbida, y matriz (árboles exóticos y pastizal natural; Figura 4). En la localidad de Pajcha se observó que, en su mayoría, los individuos presentaron un mayor porcentaje de árboles de $P$. subtusalbida (Figura 2). En contraste, en la localidad de San Miguel la presencia tanto de árboles de P. subtusalbida como de matriz natural de pastizal fue similar (Figura 3), e incluso se obtuvo el registro de la hembra H10 (Figura 3) que presentó un $80 \%$ de matriz natural de pastizales dentro su ámbito hogareño.

En las comparaciones de área del ámbito hogareño entre las localidades de Pajcha y San Miguel no se encontraron diferencias significativas $(F=0.078, \mathrm{~d}$. $\mathrm{f} .=1, P=$ 0.784). Sin embargo, sí se encontraron diferencias significativas al comparar el porcentaje de presencia de árboles de P. subtusalbida y la matriz ( $\mathrm{W}=0.5, \mathrm{p}=0.0006639)$.

\section{Discusión}

Los efectos de especies forestales introducidas en los bosques nativos de $P$. subtusalbida han sido estudiados en los últimos años (Aguirre et al. 2004; Martínez et al. 2006; Aguirre et al. 2007; Gareca et al. 2007; Solís et al. 2014). Gareca y Martínez (2015) encontraron que las especies introducidas afectan en la reproducción y regeneración de $P$. subtusalbida, y este efecto

Tabla 1. Áreas y perímetros de los ámbitos hogareños de Phyllotis osilae. Hembra (H), macho (M).

Código N. fixed points N. noches Àrea $\left(m^{2}\right)$ MCP $95 \%$ Perímetro $(\mathrm{m}) \mathrm{MCP} 95 \% \quad$ Área $\left(\mathrm{m}^{2}\right)$ Kernel $95 \%$

\section{Localida de Pajcha}

\begin{tabular}{|c|c|c|c|c|c|c|}
\hline $\mathrm{H} 1$ & 46 & 10 & $1,035.5$ & 140.58 & $1,068.72$ & 270.61 \\
\hline $\mathrm{H} 2$ & 63 & 10 & 3,010 & 207.70 & $2,271.65$ & 426.41 \\
\hline $\mathrm{H} 3$ & 35 & 10 & 1,502 & 155.78 & - & - \\
\hline $\mathrm{H} 4$ & 45 & 10 & 1,491 & 156.18 & $1,777.89$ & 297.11 \\
\hline H5 & 19 & 9 & 50.5 & 34.27 & - & - \\
\hline H6 & 18 & 9 & 255 & 70.73 & - & - \\
\hline $\mathrm{H} 7$ & 12 & 10 & 213.5 & 58.76 & - & - \\
\hline M8 & 7 & 2 & 177.5 & 66.31 & - & - \\
\hline \multicolumn{7}{|c|}{ Localidad de San Miguel } \\
\hline H9 & 39 & 8 & 847.5 & 144.88 & 46.12 & 136.78 \\
\hline $\mathrm{H} 10$ & 43 & 8 & 2,450 & 245.68 & 37.75 & 136.69 \\
\hline M11 & 38 & 8 & 1,191 & 132.06 & - & - \\
\hline M12 & 63 & 8 & 1,274 & 182.99 & 50.45 & 142.12 \\
\hline $\mathrm{H} 13$ & 20 & 9 & 480 & 88.95 & - & - \\
\hline M14 & 31 & 6 & 1,668 & 191.92 & - & - \\
\hline $\mathrm{H} 16$ & 30 & 9 & 736 & 112.57 & - & - \\
\hline $\mathrm{H} 17$ & 32 & 10 & 499 & 87.22 & 971.09 & 169.37 \\
\hline
\end{tabular}



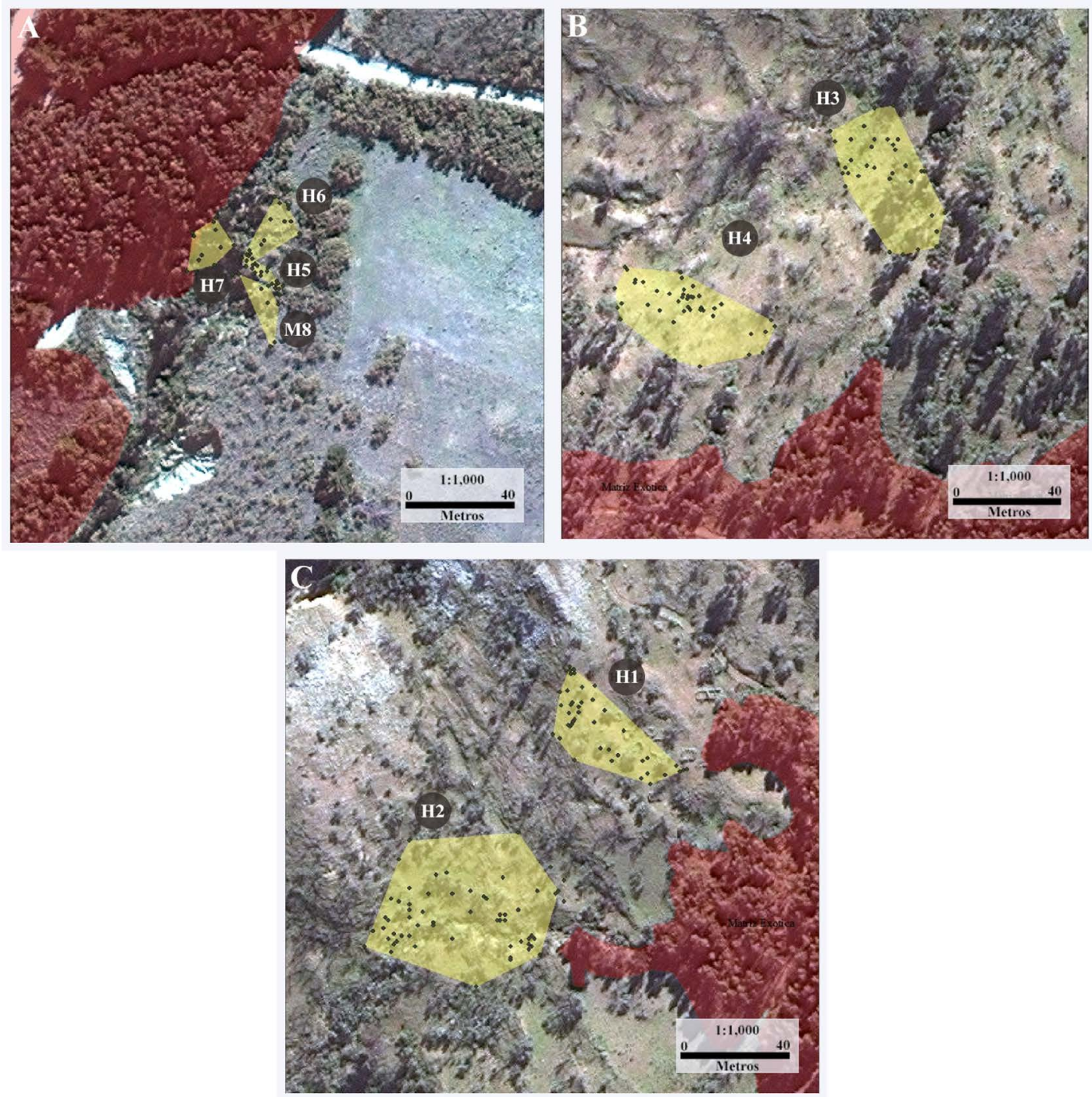

Figura 3. Ámbitos hogareños (polígonos amarillos) de la localidad de Pajcha con matriz de plantaciones de árboles exóticos (polígono rojo). A): Fragmento PAJ2, individuos H5, H6, $\mathrm{H} 7$ y M8. B): Fragmento PAJ1, individuos $\mathrm{H} 4$ y H3. C): Fragmento PAJ1, individuos $\mathrm{H} 2$ y H1.

puede diferir según la localidad y el tipo de bosque. La matriz de árboles exóticos igualmente tendría un efecto sobre los pequeños mamíferos como $P$. osilae. Nuestros resultados de ámbito hogareño muestran que la matriz de árboles exóticos no tendría un efecto sobre el área o perímetro, pero si sobre su movilidad; esto se evidencia en la localidad de Pajcha, con la ausencia de movimientos de $P$. osilae de los fragmentos a la matriz exótica (Figura 2). Kupfer et al. (2006) mencionan que una matriz opera como una barrera, filtro o conducto que variará según el tipo de perturbación, en este caso, la matriz de árboles exóticos estaría operando como una barrera. La presencia de plantaciones forestales con plantas exóticas ocasiona impactos sobre los acuíferos y perturban gravemente el ciclo de nutrientes en los suelos debido a la resistencia a la descomposición de la materia orgánica muerta (Navarro 2015). Esto se ve reflejado en la ausencia casi total de matriz de árboles exóticos en los ámbitos hogareños, a excepción del individuo H7 (Figura 2) que presenta un $33 \%$ de matriz exótica. Por otro lado, la matriz natural de pastizal tiene un rol importante en los ámbitos hogareños de $P$. osilae. Patton et al. (2015) indican que esta especie está muy restringida a los hábitats de pasto dominado por el género
Stipa en el Altiplano. La composición florística de localidad de San Miguel incluye gramíneas de este género (Stipa ichu), permitiendo la presencia de matriz natural de $P$. osilae, y en un caso $\mathrm{H} 10$ (Figura 3), el porcentaje de esta matriz es mayor que el fragmento.

Las diferencias entre los ámbitos hogareños de machos $y$ hembras son comunes (Sanches et al. 2012). En ratones una característica típica es que los machos se sobrepongan con los de varias hembras (Bonatto et al. 2013). Bond y Wolf (1999) demostraron que en roedores microtinos (Microtus canicaudus) el tamaño del ámbito hogareño de los machos esta influenciado por una combinación de la densidad, acceso a las hembras y competencia con los machos. Bonnatto et al. (2012) determinaron en el ámbito hogareño de Akodon azarae los machos se evitan unos de otros, esta evasión espacial reflejaría la competencia entre los machos por monopolizar a las hembras receptivas, haciéndolos más vulnerables a ser depredados, razón por la cual se capturaron pocos individuos machos $(n=4)$. Los ámbitos hogareños de la localidad de Pajcha están conformados en su mayoría por hembras ( $n=6$; Figura 2$)$, donde se observa que el $100 \%$ de presencia de árboles 

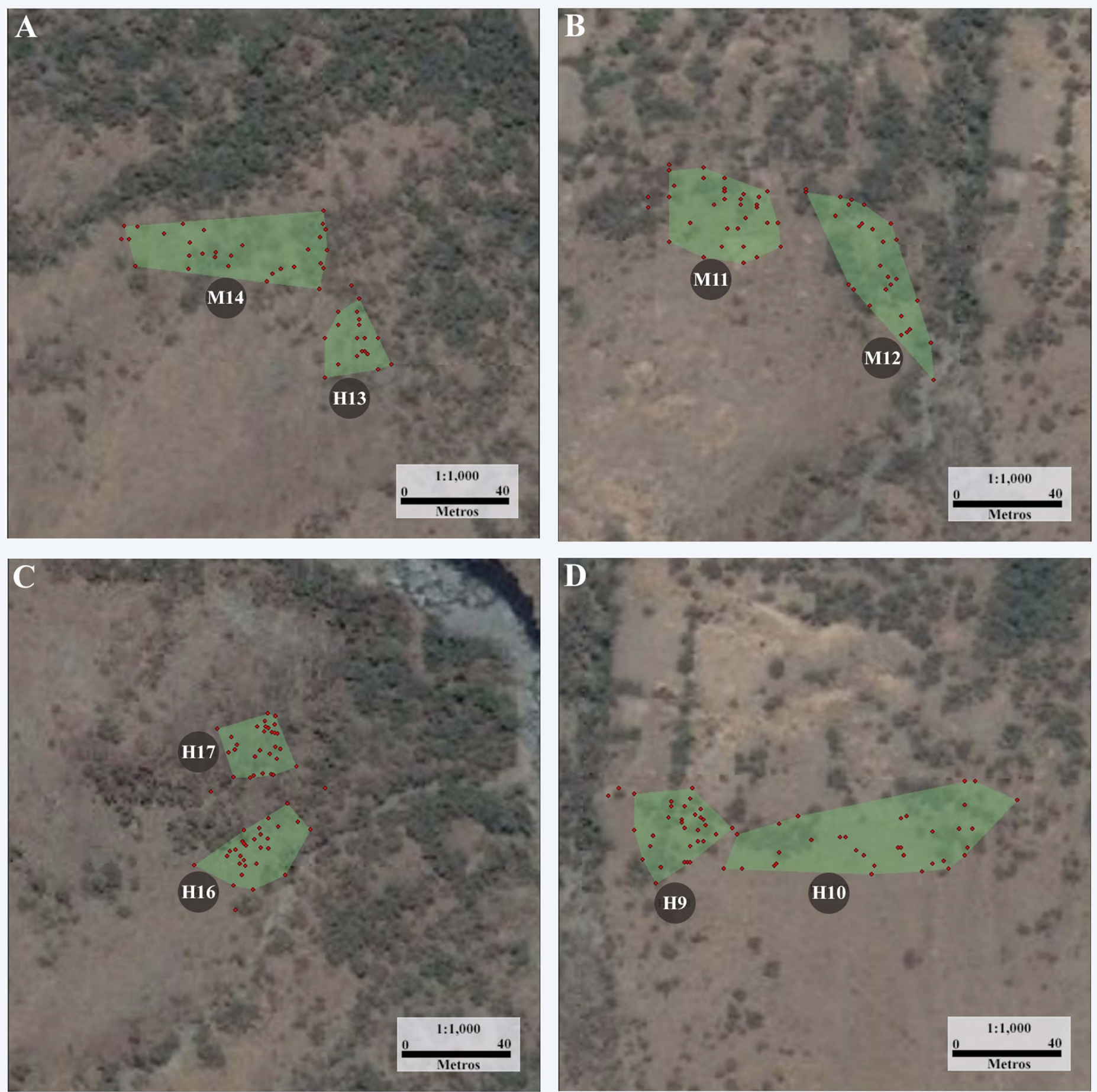

Figura 3. Ambitos hogareños (polígonos verdes) de la localidad de San Miguel con matriz de pastizales. A): Fragmento SM11, individuos M14 y H13. B): Fragmento SM11, individuos M11 y M12. C): Fragmento SM13, individuos H17 y H16. D): Fragmento SM11, individuos H9 y H10.

de $P$. subtusalbida, esto indicaría que los fragmentos de esta especie es un hábitat con abundantes recursos para pequeños mamíferos como $P$. osilae.

La generación de información sobre aspectos de la reproducción de Polylepis podría ayudar, en alguna medida, a remediar el problema de la fragmentación de estos bosques (Vega et al. 2018). La dispersión de semillas por el viento puede predominar en algunas especies de Polylepis (Simpson 1979), pero la superficie de los frutos de diferentes especies tiene crestas, bultos, espinas o alas, lo que también sugiere la dispersión animal (Simpson 1986). Balderrama (2013, com. pers.) encontraron que P. osilae consume semillas de $P$. subtusalbida, además, determinaron el grado de daño causado a las semillas y registraron que el consumo parcial sería la principal forma de P. osilae para consumir semillas. También registraron que en fragmentos de bosques de $P$. subtusalbida con matriz de pastizal natural y plantaciones de árboles exóticos, $P$. osilae es la especie dominante en la estructura de la comunidad de roedores. Conocer aspectos de la biología de Polylepis, así como sus interacciones planta-animal son de mucha importancia, ya que roedores como $P$. osilae pueden estar incrementando la probabilidad de germinación de semillas y permitiendo la distribución de semillas hacia lugares donde la vegetación 


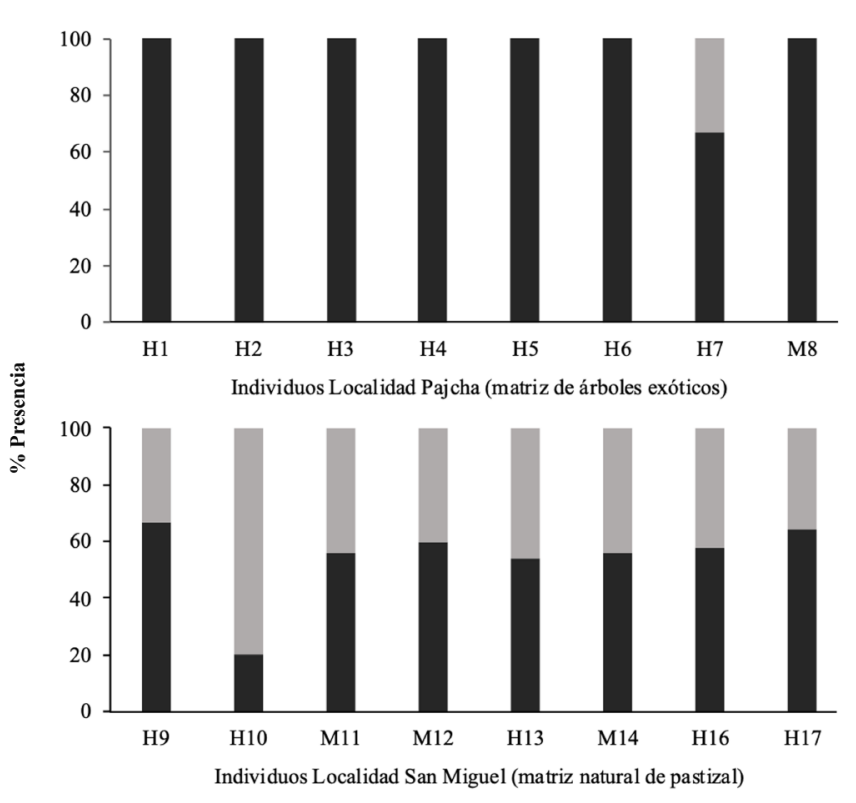

Figura 4. Porcentajes de presencia de Polylepis subtusalbida (color negro) y matriz (color gris) dentro los ámbitos hogareños de Phyllotis osiale.

natural ha sido removida, cumpliendo un rol importante en la recuperación y conservación de estos ecosistemas. Por lo anterior, conocer aspectos de su historia natural como su ámbito hogareño es de mucha importancia, al igual que sus respuestas a la fragmentación para predecir su propio destino y el de los bosques de Polylepis.

\section{Agradecimientos}

El trabajo fue realizado dentro el proyecto "Permeabilidad de la Matriz para el Flujo de Genes en Bosques Altoandinos Amenazados de Polylepis" del Centro de Biodiversidad y Genética de la Universidad Mayor de San Simón, financiado por la cooperación belga VLIR-IUC. Deseamos agradecer a O. Osco, P. Mejía, M. Peñaranda, L. Cáceres y S. Avilés por la colaboración en el trabajo de campo, igualmente a R. Vargas, J. Salazar, J. C. Pérez, D. Peñaranda y L. Siles. También deseamos agradecer a L. Guevara y a los dos revisores anónimos que mejoraron el manuscrito con sus comentarios. Igualmente deseamos dedicar este trabajo al Dr. Sydney Anderson quien ha sido un pilar fundamental en la mastozoología contemporánea boliviana.

\section{Literatura citada}

Aguirre, L. F., O. Ruiz, A. Lisperguer, R. Quinteros, L. Paz-Soldán, Y D. BARJA. 2004. Biodiversidad y comunidades animales en bosques fragmentados andinos. Ciencia y Tecnología 3:7-10.

Aguirre, L. F., J. A. Balderrama, C. F. Pinto, E. I. Maradiegue, Y R. VArGas. 2007. Influencia de dos especies forestales exóticas sobre fauna terrestre de bosques nativos de kewiña en el Parque Nacional Tunari Pp. 1420-1424 in Congreso Internacional sobre Desarrollo, Medio Ambiente y Recursos Naturales: Sostenibilidad a múltiplos niveles y escalas Vol. III. (Feyen, J., L. F. Aguirre, y M. Moraes, eds.). Cochabamba, Bolivia.

Anderson, S. 1997. Mammals of Bolivia, taxonomy and distribution. Bulletin of the American Museum of Natural History 231:1-652.
Arrázola, S. 2011. Polylepis subtusalbida. Pp. 62-64 in Libro Rojo de la Flora amenazada de Bolivia. Vol. I. Zona Andina (Ministerio de Medio Ambiente y Agua). La Paz, Bolivia.

Bonatto F., D. Gomez, A. Steinmann, y J. Priotto. 2012. Mating strategies of Pampean mouse males, Animal Biology 62:381-396.

Bonatto, F., J. Coda, D. Gomez, J. Priotto, y A. Steinmann. 2013. Inter-male aggression with regard to polygynous mating system in Pampean grassland mouse, Akodon azarae (Cricetidae: Sigmodontinae). Journal of Ethology 31:223-231.

Bond, M. L., Y J. O. Wolff. 1999. Does Access to Females or Competition among Males Limit Male Home-Range Size in a Promiscuous Rodent? Journal of Mammalogy 80:1243-1250.

Burt, W. 1943. Territoriality and Home Range Concepts as Applied to Mammals. Journal of Mammalogy 24:346-352.

Cumming, G. S., y D. Cornélis. 2012. Quantitative comparison and selection of home range metrics for telemetry data. Diversity and Distributions 18:1057-1065

Delciellos, A. C., C. dos Santos de Barros, J. A. Prevedello, M. S. Ferreira, R. Cerqueira, y M. V. Vieira. 2018. Habitat fragmentation affects individual condition: evidence from small mammals of the Brazilian Atlantic Forest. Journal of Mammalogy 99:936-945.

Dressler, F., S. Ripperger, M. Hierold, T. Nowak, C. Eibel, B. Cassens, F. Mayer, K. Meyer-Wegener, y A. Kolpin. 2016. From radio telemetry to ultra-low-power sensor networks: tracking bats in the wild. Institute of Electrical and Electronics Engineers Communications Magazine 54:129-135.

Eiris, G. C., Y G. R. BARRETo. 2009. Home range of marsh rats, Holochilus sciureus, a rodent pest in rice fields of Venezuela. Interciencia 34:400-405.

FJeLdSÅ, J., Y M. KessLer. 2004. Conservación de la biodiversidad en los bosques de Polylepis de tierras altas de Bolivia. Una contribución al manejo sustentable en los Andes, DIVA Technical Report 11, Editorial FAN. Santa Cruz de la Sierra, Bolivia.

Forman, R. T. T. 1995. Land Mosaics: The Ecology of Landscapes and Regions. Cambridge University Press. Cambridge, Reino Unido.

Gareca, E., Y. Y. Martínez, R. O. Bustamante, L. F. Aguirre, y M. M. SILEs. 2007. Regeneration patterns of Polylepis subtusalbida growing with the exotic trees Pinus radiata and Eucalyptus globulus at Parque Nacional Tunari, Bolivia. Plant Ecology 193:253-263.

Gareca, E., y Y. Y. Martínez. 2015. Árboles introducidos y nativos conviviendo en el Parque Nacional Tunari - ¿Tienen problemas? Pp. 220-223 in Biodiversidad, Ecología y Conservación del Valle Central de Cochabamba. Centro de Biodiversidad y Genética (CBG), Universidad Mayor de San Simón. Cochabamba, Bolivia.

Hammer, Ø., D. A. Harper, y P. D. Ryan. 2001. PAST: Paleontological statistics software package for education and data analysis. Palaeontologia electronica 4: 9.

Harris, S., W. J. Cresswell, P. G. Forde, W. J. Trewhella, T. Woollard, y S. WRAY. 1990. Home-range analysis using radio-tracking data-a review of problems and techniques particularly as applied to the study of mammals. Mammal Review 20:97-123.

Hershrovitz, P. 1962. Evolution of Neotropical Cricetine Rodents (Muridae) with special reference to the Phyllotine group. Fieldiana, Zoology 46:1-524. 
IBISCH, P. L., y G MéRIDA (eds.). 2003. Biodiversidad: La Riqueza de Bolivia, Estado de Conocimiento y Conservación. Ministerio de Desarrollo Rural, Agropecuario y Medio Ambiente. Editorial FAN. Santa Cruz de la Sierra, Bolivia.

JAYAT, J. P., y S. PACheCo. 2006. Distribución de Necromys Lactens y Phyllotis osilae (Rodentia: Cricetidae: Sigmodontinae) en el noroeste argentino: Modelos predictivos basados en el concepto de nicho ecológico. Mastozoología Neotropical 13:69-88.

JoRge, M. L. S. P. 2008. Effects of forest fragmentation on two sister genera of Amazonian rodents (Myoprocta acouchi and Dasyprocta leporina). Biological Conservation 141:617-623.

Kupfer, J. A., G. P. Malanson, y S. B. Franklin. 2006. Not seeing the ocean for the islands: the mediating influence of matrixbased processes on forest fragmentation effects. Global Ecology and Biogeography 15:8-20.

Martínez, Y. Y., E. Gareca, L. Meneses, F. R. Castro, y L. F. Aguirre. 2006. Cambios en la morfología de frutos y germinación de semillas de Polylepis besseri por interacciones con especies exóticas en el Parque Nacional Tunari. Pp. 55 in Libro de resúmenes II Congreso internacional de ecología y conservación de bosques de Polylepis (Ojeda P., M. Guardamiño, C. Aucca, J. Cahill, y H. Arnal, eds.). Centro de Biodiversidad y Genética, UMSS Bolivia y American Bird Conservancy. Lima, Perú.

Ministerio de Medio Ambiente, Agua y Cambio Climático. 2011. Libro Rojo de la Flora amenazada de Bolivia. Vol. I. Zona Andina. La Paz, Bolivia.

Moнr, C. O. 1947. Table of equivalent populations of North American small mammals. American Midland Naturalist 37:223-249.

Navarro, G. 2015. Ecosistemas altimontanos de la Cordillera del Tunari, Geobotánica. Pp. 178-194 in Biodiversidad, Ecología y Conservación del Valle Central de Cochabamba. (Navarro, G., L. F. Aguirre, y M. Maldonado, eds.)Centro de Biodiversidad y Genética (CBG), Universidad Mayor de San Simón. Cochabamba, Bolivia.

Navarro, G., L. F. Aguirre, y M. Maldonado (eds.). 2015. Biodiversidad, Ecología y Conservación del Valle Central de Cochabamba. Centro de Biodiversidad y Genética (CBG), Universidad Mayor de San Simón. Cochabamba, Bolivia.

Ostfeld, R. S. 1985. Limiting resources and territoriality in microtine rodents. The American Naturalist 126:1-15.

Patton, J. L., U. F. J. PardiñAs, y G. D'Elía (eds.). 2015. Mammals of South America, Volume 2 Rodents. The University of Chicago Press. Chicago, EE.UU.

Prevedello, J. A., y M. V. Vieira. 2010. Does the type of matrix matter? A quantitative review of the evidence. Biodiversity and Conservation 19:1205-1223.

Ruiz, O., L. F. Aguirre, R. Vargas, R. Aguayo, F. Alfaro, y K. Moya. 2004. Protocolo de investigación para la fauna altoandina del Parque Nacional Tunari, Parte I: Mamíferos, Anfibios y Reptiles. Documentos en Biodiversidad y Conservación 1:1-17.

Sanches, V. Q. A., M. M. de Arruda Gomes, F. de Camargo Passos, G. Graciolli, y A. Cesar de Aquino Ribas. 2012. Home-range and space use by Didelphis albiventris (Lund 1840) (Marsupialia, Didelphidae) in Mutum Island, Paraná river, Brazil. Biota Neotropica 12:1-6.
Santini, L., M. Di Marco, P. Viscontia, D. Baisero, L. Boitani, y C. Rondinini. 2013. Ecological correlates of dispersal distance in terrestrial mammals. Hystrix 24:181-186.

Sikes, R. S., W. l. Gannon, y The Animal Care and Use Committee of the American Society of Mammalogists. 2011. Guidelines of the American Society of Mammalogists for the use of wild mammals in research. Journal of Mammalogy 92:235-253.

Simpson, B. B. 1979. A revision of the genus Polylepis (Rosaceae: Sanguisorbeae). Smithsonian Contributions to Botany 43:1-62.

Simpson, B. B. 1986. Speciation and specialization of Polylepis in the Andes. Pp. 304-316 in High altitude tropical biogeography (Vuillemier, F., y M. Monasterio, eds.). Oxford University Press \& American Museum of Natural History. New York, EE. UU.

Solís, C., Y. Y. Martínez y E. Gareca. 2014. Relación entre las condiciones del origen de las semillas y la germinación de Polylepis subtusalbida en vivero. Pp. 161 in Memorias del IV Congreso de ecología en Bolivia (Mostacedo, B., D. Villarroel, M. Toledo, J. Pinto, G. Carreño-Rocabado, B. Flores, Y. V. Usiar, eds.). Universidad Autónoma Gabriel René Moreno. Santa Cruz, Bolivia.

Spotorno, A. E., J. P. Valladares, J. C. Marin, R E. Palma, y C. Zuleta. 2004. Molecular divergent and phylogenetic relationships of Chinchillids (Rodentia: Chinchillidae). Journal of Mammalogy 85:384-388.

Steppan, S. J. 1995. Revision of the Tribe Phyllotini (Rodentia: Sigmodontinae), with a phylogenetic hipótesis for the Sigmodontinae. Fieldiana Zoology 1464:1-112.

Tarifa, T., y E. Yensen. 2001. Mammals of Bolivian Polylepis woodlands. Revista Boliviana de Ecología y Conservación Ambiental 9:29-44.

Tarifa, T., y L. F. Aguirre. 2009. Capitulo 6. Mamíferos. Pp. 419 572 in el Libro rojo de la fauna silvestre de vertebrados de Bolivia (Ministerio de Medio Ambiente y Agua) La Paz, Bolivia.

Vargas, R., E. I. Maradiegue, y L. F. Aguirre. 2007. Pequeños mamíferos de bosques nativos y plantaciones forestales exóticas en el Parque Nacional Tunari, Cochabamba, Bolivia Pp. 1374-1381 in Congreso Internacional sobre Desarrollo, Medio Ambiente y Recursos Naturales: Sostenibilidad a múltiplos niveles y escalas Vol. III. (Feyen, J., L. F. Aguirre, y M. Moraes, eds.). Cochabamba, Bolivia.

Vega, C. K., C. G. Villegas, P. A. Rocabado, J. A. N. Quezada, M. Y. López, y A. W. Quevedo. 2018. Biología reproductiva de tres especies de Polylepis ( $P$. neglecta, $P$. incarum y $P$. pacensis), con énfasis en su comportamiento germinativo. Ecología Austral 28:310-324.

White G. C., y R. A. Garrott. 1990. Analysis of Wildlife RadioTracking Data, Academic Press. California, EE.UU.

Wood, B. A., L. CaO, y M. D. Dearing. 2010. Deer mouse (Peromyscus maniculatus) home-range size and fidelity in sage-steppe habitat. Western North American Naturalist 70:345-354.

Worton, B. J. 1989. Kernel methods for estimating the utilization distribution in home-range studies. Ecology 70:164-168.

Yensen, E., y T. Tarifa. 2002. Mammals of Bolivian Polylepis woodlands: guild structure and diversity patterns in the world's highest woodlands. Ecotropica 8:145-162. 
Associated editor: Lazaro Guevara

Submitted: May 20, 2020; Reviewed: July 28, 2020;

Accepted:August 11, 2020; Published on line: September 17, 2020. 